\title{
Development and management of a periapical lesion in a known case of systemic lupus erythematosus - a
} case report

\begin{abstract}
Objective: The aim of this article is to report a case of systemic lupus erythematosus (SLE) under treatment with sudden and unexplained development of periapical periodontitis in mandibular central incisors. Clinical Presentation and Intervention: The patient was a 24 year old girl with a known history of systemic lupus erythematosus (SLE) and was receiving immunosuppressant therapy including steroids since four years. She reported that her lower anterior teeth had continuous moderate pain for the last one week. She was diagnosed as having periapical periodontitis and treated as required.
\end{abstract}

Conclusion: Patients with systemic lupus erythematosus receiving immunosuppressant therapy especially steroids could be at a risk of development of apical radicular lesions advocating endodontic intervention. It is therefore recommended that patients of SLE should have regular dental checkups at least 6 monthly.

Keywords: systemic lupus erythematosus, steroids, immunosuppressant therapy, periapical periodontitis
Volume 6 Issue I - 2017

\section{Farhat Amin,' Salman Aziz ${ }^{2}$}

'Department of Orthodontics College of Dentistry, King Khalid University, Saudi Arabia

${ }^{2}$ Department of Dentistry, Al Jouf University, Saudi Arabia

Correspondence: Salman Aziz, Department of Dentistry, College of Dentistry, Al Jouf University, Saudi Arabia, Emaildrsalmanaziz@gmail.com

Received: July 26, 2016 | Published: January 11, 2017

\section{Introduction}

Systemic lupus erythematosus (SLE) is an autoimmune connective tissue disease that may affect any part of the body. The body's cells and tissues are attacked by immune system, resulting in inflammation and tissue damage. ${ }^{1}$ SLE is a type III hypersensitivity reaction caused by the formation of antibody-immune complexes. The most often affected organs and body parts are joints, heart, skin, lungs, blood vessels, kidneys, liver, and nervous system. The disease follows an unpredictable course, with periods of flares alternating with remissions. SLE is nine times more common in women than men. Women of child-bearing ages i.e. 15 to 35 years especially those of non European decent are most often affected. ${ }^{2-4}$ SLE is usually treated utilizing immunosuppression. Cyclophosphamide, corticosteroids and other Immunosuppressants are utilized. There is currently no aliment. SLE can be fatal; however with recent advances in the field of medicine, fatalities are becoming rare. Survival for patients with SLE in Canada, Europe and United States has risen to approximately, $78 \%$ at 20 years, $90 \%$ at 10 years, and $95 \%$ at five years 4 and now approaches that of matched controls without lupus. The aim of this report was to highlighten the occurrence of dental problems among patients of SLE. Clinical Presentation and Intervention.

\section{Chief complaint}

A 24-year-old Pakistani girl presented with chief complaint of moderate pain in anterior area of lower arch since last one week.

\section{Past medical history}

She gave a history of systemic lupus erythematosus and hypothyroidism since last four years. She mentioned problems like immense hair fall, oral ulcers, stomach ache, diarrhea and fever since that time. She was taking Deltacotril, Plaqunil, Cellcept and Thyroxin. She was tapered on these medicine two years back, after which she got a bad flare on SLE. It was an attack of lupus nephritis and she had to go for a kidney biopsy. She had to consult a rheumatologist too after every 2-3 months.

\section{Past dental history}

Patient received orthodontic treatment for couple of years in adolescent for correction of malocclusion.

\section{Extra- oral examination}

The frontal view showed no facial asymmetry. The patient had a slight convex lateral profile and a normal nasolabial angle with competent lips (Figure 1A \& 1B).

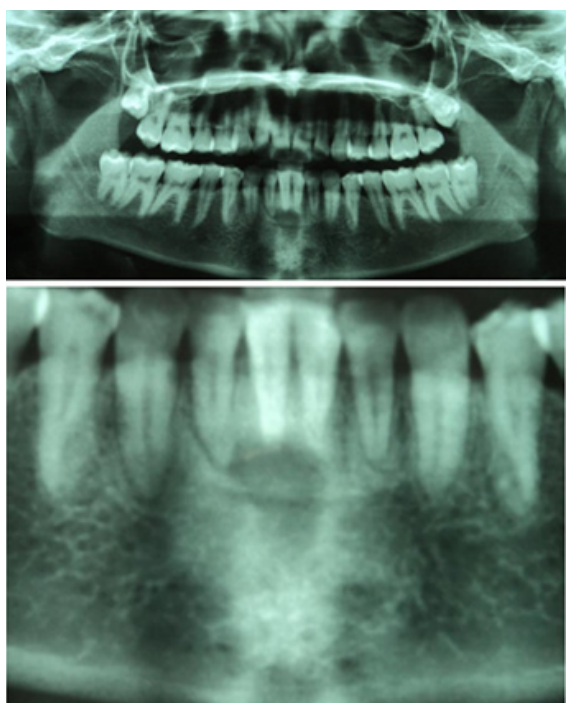

Figure IA-IB Extra- oral examination. 


\section{Intra- oral examination}

The patient had a full permanent dentition apart from the third molars. The incisor relationship was class 11 division 1 with an overjet measured to the upper left central incisor of $4 \mathrm{~mm}$ and an overbite of $4 \mathrm{~mm}$. Upper and lower midlines were correctly placed with respect to the midsagital plane of the face. The lower arch was symmetrical. Mandibular central incisors \#41, 31(FDI) were mesiolingually rotated with grade 1 mobility (according to millers classification) along with swelling and tenderness to palpation at the peri apical area. The gum margins of these teeth were inflamed with low attachment of labial frenum. The upper arch was symmetrical and teeth were well aligned. There was generalized marginal gingivitis (Figure 1C \& 1D).
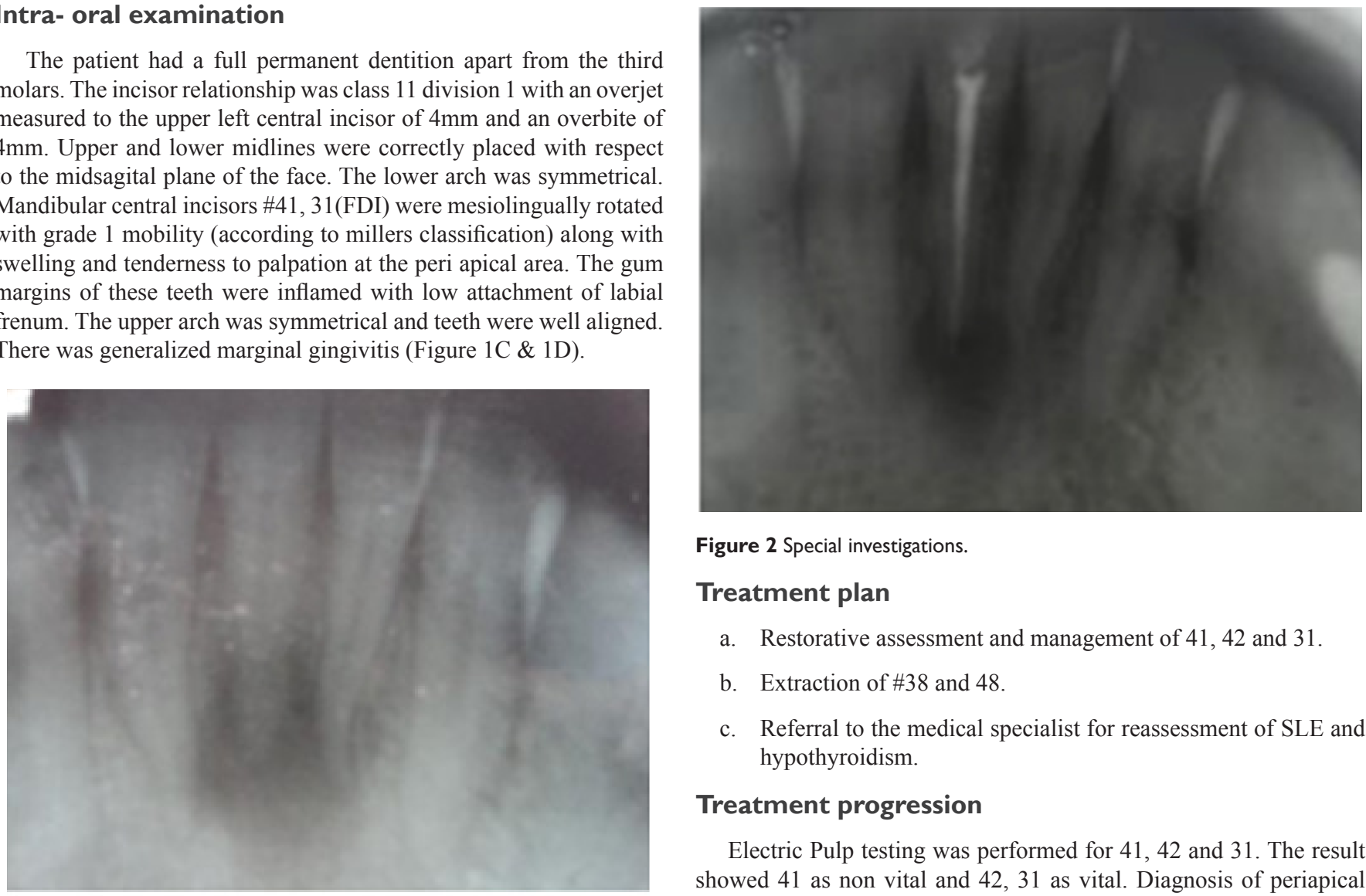

Figure 2 Special investigations.

\section{Treatment plan}

a. Restorative assessment and management of 41, 42 and 31 .

b. Extraction of \#38 and 48 .

c. Referral to the medical specialist for reassessment of SLE and hypothyroidism.

\section{Treatment progression}

Electric Pulp testing was performed for 41, 42 and 31. The result showed 41 as non vital and 42, 31 as vital. Diagnosis of periapical periodontitis was made and root canal therapy was executed on $\# 41$. Intracanal dressing of calcium hydroxide was placed between appointments, and obturation was done after confirmation of dryness of canal. Patient's symptoms subsided progressively. Pulp test was again carried out for $\# 42$ and 31 which remained vital at 6 months and are still under observation at 1 year.

\section{Discussion}

Classically, LE has been subdivided into a systemic and a cutaneous form. While systemic lupus erythematosus (SLE) is a multiorgan disease with variable prognosis, cutaneous lupus erythematosus (CLE) is a more benign condition-limited to skin and/or mucosal surfaces. ${ }^{5-7}$ The prevalence of mucosal involvement in LE patients is debatable. Some authors suggest that oral lesions are present in $9-45 \%$ of patients with the systemic form of the disease and in 3-20\% in those with CLE. ${ }^{8-10}$ Lupus are diagnosed by laboratory histological examination of biopsies; however, the presence of certain lesions and some clinical characteristics can help in an early diagnosis and improve the disease prognosis. SLE patients are generally given immunosuppressants that may lead to systemic conditions-such as suture dehiscence, increased risk of infection, and delayed healing-that deserve specific attention during dental treatment. Clinical aspects of lesions vary, and lips and buccal mucosa are the most affected sites. Histologically, lesions revealed lichenoid mucositis with perivascular infiltrate and thickening of basement membrane. ${ }^{11}$ Allis Marvis reported a case of an SLE patient with oral manifestations with ulcerative lesions in the oral cavity along with development of lupus nephritis. ${ }^{12}$ The main theme of this case report was to relate a systemic problem of a 24 year 
old girl who was diagnosed as having SLE. She received orthodontic treatment for correction of malalignment when she was an adolescent. She reported to the hospital with acute pain in lower anterior region along with mesiolingual rotation of 31 and 41 . Although there was slight blunting of apical ends of root of lower incisors which might be a consequence of orthodontic treatment which had been completed few years back. There is insufficient literature available as of yet regarding development of periapical lesions in orthodontic patients after treatment or its formation in an SLE patient as an after effect of taking immunosuppresants and systemic steroids. The second possibility seems to be less investigated and therefore must be kept in mind when evaluating patients of SLE taking steroids. Steroids and Immunosuppressants like cyclophosphamide have been known to decrease immunity thereby rendering a person prone to diseases and periapical periodontitis is one of such diseases. A chance of development of periapical lesion is also present in patients with poor oral hygiene, which can be in the form of apical cyst of any involved tooth. Such lesions are sometimes difficult to diagnose. They are sometimes misdiagnosed and the importance of a differential diagnosis has to be noted. ${ }^{13}$ Periapical cemental dysplasia is known to be related to periapical granuloma. ${ }^{14,15}$ Giant cementoblastoma can also be misdiagnosed as an endodontic lesion. ${ }^{16-19}$ Paradental $^{20}$ and Stafne's bone cavity, ${ }^{21}$ have also been reported. We need to be careful in the diagnosis of these diseases, because they sometimes manifest similar symptoms to those of a periapical lesion. To prevent misdiagnosis, application of electric pulp test and confirmation of dental history are recommended.

\section{Conclusion}

The orthodontist as a member of a multidisciplinary team that deals with almost all types of problems such as endodontic, periodontic, restorative and medical problems in their orthodontic patients. Therefore, they should have a vision and knowledge of these related problems for better evaluation and discussion with concerned specialists for proper management of these types of cases.

\section{Acknowledgments}

None.

\section{Funding}

None.

\section{Conflicts of interest}

The authors declare there is no conflict of interests.

\section{References}

1. William D James, Berger Elston. Andrews' diseases of the skin: clinica dermatology. 11th ed. St. Louis, Missouri, USA: Saunders; 2005:1-968.

2. Anisur Rahman, David A, Isenberg. Review article: systemic lupus erythematosus. N Engl J Med. 2008;(9):929-939.
3. Lupus foundation of America.

4. Harrison's Internal Medicine. 17th ed. Systemic lupus erythematosus.

5. Crowson NA, Magro C. The cutaneous pathology of lupus erythematosus: a review. J Cutan Pathol. 2001;28(1):1-23.

6. Dubois EL. Lupus erythematosus: A review of the current status of discoid and systemic lupus erythematosus and their variants. Los Angeles, California, USA: University of Southern California Press; 1976.

7. Karjalainen TK, Tomich CE. A histopathologic study of oral mucosal lupus erythematosus. Oral Surg Oral Med Oral Pathol. 1989;67(5):547554.

8. Burge SM, Frith PA, Juniper RP, et al. Mucosal involvement in systemic and chronic cutaneous lupus erythematosus. $\mathrm{Br} J$ Dermatol. 1989;121(6):727-741.

9. Jonsson R, Hieden G, Westberg NG, et al. Oral mucosal lesions in systemic lupus erythematosus: a clinical, istopathological and immunopathological study. J Rheumatol. 1984;11(1):38-42.

10. Schiodt M. Oral discoid lupus erythematosus. III. A histopathologic study of sixty-six patients. Oral Surg Oral Med Oral Pathol. 1984;57(3):281293.

11. Silvia V Lourencxo, Fabio R, Boggio P, et al. Lupus erythematosus: clinical and histopathological study of oral manifestations and immunohistochemical profile of the inflammatory infiltrate. $J$ Cutan Pathol. 2007;34(7):558-564.

12. Allais Marvis, Sverzut AT, Mazzonetto R, et al. Oral manifestation of systemic LE: Lupus nephritis - a case report. Gen Dent. 2008;56(1):35-41.

13. Benatti BB, Carvalho MD, Games BP. Importance of differential diagnosis in endodontic-periodontic lesions: case reports. Gen Dent. 2003;51(3):246-248.

14. Ward MR. Periapical cemental dysplasia: a case report. New Zeal Dent J. 1993;89(395):53-54.

15. Smith S, Patel K, Hoskinson AE. Periapical cemental dysplasia: a case of misdiagnosis. Br Dent J. 1998;185(3):122-123.

16. Putenman M, Fliss DM. Giant cementoblastoma simulating a periodontal infection. J Laryngal Otal. 1988;102(3):264-266.

17. Silva TA, Batista AC, Camanini. Paradental cyst mimiking a radicular cyst on the adjacent tooth: case report and review of terminology. $J$ Endod. 2003;29(1):73-76.

18. Fregnani ER, De Moraes Romas FM, Nadalin MR. Simple bone cyst: possible misdiagnosis in periapical pathology. Gen Dent. 2007;55(2):129131.

19. De Maraes Romas Perez FM, Soares UN, Silva Sousa, et al. Ossifying fibroma misdiagnosed as chronic apical periodontitis. $J$ Endod. 2010;36(3):546-548.

20. Golgano C, Samson J, Kuffer R. Focal cemento-osseous dysplasia involving a mandibular lateral incisor. Int Endod J. 2003;36(12):907-911.

21. Bonnstein MM, Wiest R, Balsiger R. Anterior Stafne's bone cavity mimiking a periapical lesion of endodontic origion: report of two cases. $J$ Endod. 2009;35(11):1598-1602. 